\title{
On the Function and Limitation of the Arbitrariness of Saussure's Linguistic Signs
}

\author{
Ying Zhou ${ }^{1}$, Jiugen Xiao'
}

School of Chinese Language and Literature, Research Center of Language and Language Life, Jiangxi Normal University, Nanchang, China

\section{Email address:}

992963813@qq.com (Ying Zhou),jxsdxjg666666@sina.com (Jiugen Xiao)

\section{To cite this article:}

Ying Zhou, Jiugen Xiao. On the Function and Limitation of the Arbitrariness of Saussure's Linguistic Signs. Science Innovation. Vol. 5, No. 5, 2017, pp. 289-292. doi: 10.11648/j.si.20170505.18

Received: June 10, 2017; Accepted: July 11, 2017; Published: August 2, 2017

\begin{abstract}
Saussure's general language course is the first masterpiece of modern linguistics. One of the important contents is the arbitrariness of linguistic signs. In this regard, more and more scholars questioned that language signs are iconicity or motivation, iconicity and arbitrariness in opposition, pointed out that Saussure discussed the arbitrariness of language symbol have great limitations, regardless of the signifier and signified, or its elements (pronunciation, vocabulary, syntax), There is a non - arbitrariness; and that, even if Saussure proposed absolute arbitrariness and relative arbitrariness theory, some problems still can't explain. In our opinion, everything in the world is relative, and we should also take a dialectical approach to the arbitrariness of linguistic signs.
\end{abstract}

Keywords: Saussure, Linguistic Signs, Arbitrariness, Function, Limitation

\section{略论索绪尔语言符号任意性的作用及局限}

周颖 ${ }^{1}$, 肖九根 ${ }^{2}$

江西师范大学文学院, 语言与语言生活研究中心, 南昌, 中国

邮箱

992963813@qq.com（周颖）, jxsdxjg666666@sina.com（肖九根）

摘要：索绪尔的《普通语言教程》是现代语言学开山之作, 其中一个重要内容就是语言符号的任意性。对此, 越来越 多的学者提出质疑, 认为语言符号具有象似性或理据性, 并将象似性与任意性对立起来, 指出索绪尔所论述的语言符 号任意性存有很大局限性, 不论其能指、所指, 还是其要素（即语音、词汇、句法）都存在着非任意性; 并且认为, 即使索绪尔提出了绝对任意性和相对任意性理论, 有些问题依然解释不清。我们认为, 世界上任何事物都是相对的, 对于语言符号任意性问题，我们也同样应辩证地看待。

关键词：索绪尔，语言符号，任意性，作用，局限

\section{1. 引言}

索绪尔不仅是结构主义创始人, 还是现代语言学的奠 基者, 美国描写语言学创建人布龙菲尔德在评论萨丕尔 《语言论》时赞扬索绪尔为“语言学研究的新方向奠定了 理论基础”。[1]（p317-319）正因为这样, 索绪尔的《普 通语言学教程》也成为现代语言学的开山奠基之作, 在语 言学史上占有十分重要的地位。在《普通语言学教程》中, 索绪尔就语言问题提出了许多新的理论观点, 而语言符号 的任意性就是其中一个非常重要的观点。 
随着索绪尔理论研究的深入, 人们对其理论观点的质 疑也日益增多, 如他的语言符号任意性观点一直以来就成 为学术界争论的焦点。在国外, 以乔姆斯基为代表的形式 主义语言学派将索绪尔的语言符号任意性作了深度发挥; 而雅库宾斯基则首先发难, 他批评索绪尔语言符号任意性 的观点, 提出了语言本质论的社会观。[2]此后, 以认知语 言学派为代表的一批学者对语言符号任意性都持不同意 见。[3]在我国, 大多数学者如陈望道、高名凯等认同索绪 尔的观点, 而许国璋、沈家煊、王寅、李葆嘉等对其语言 符号任意性的内涵、能指、所指等方面则提出了质疑。质 疑者认为语言是非任意性、象似性或理据性的。但不可否 认, 长期以来, 索绪尔语言符号的任意性对整个语言学界 产生了重大而深远的影响。

\section{2. 语言符号任意性理论的历史作用与现实意义}

\section{1. 任意性理论的历史作用}

索绪尔之前, 柏拉图明确反对事物的任意性, 他认为 任何人可以任意地给事物命名是不正确的。他的学生亚里 士多德也认为: “任何语言的声音与所指事物之间没有任 何自然联系。”[3]我国战国时期思想家、教育家荷子在其 《正名篇》中提出: “名无固宜, 约之以命, 约定俗成谓 之宜, 异于约则谓之不宜。名无固实, 约之以命实, 约定 俗成谓之实名。”也就是说, 事物的命名无所谓合理不合 理, 只要人们共同约定就行了。约定俗成就是合理的, 不 合于约定俗成的就是不合理的。约定俗成后, 就是名实相 符了。毫无疑问, 索绪尔提出语言符号任意性是对先前学 者理论的一次彻底颠覆, 这在语言学、符号学甚至许多学 科领域里都掀起了惊天骇浪。虽然索绪尔提出语言符号任 意性之前, 已有学者提及过这种观点, 但唯有索绪尔以其 丰富的学识, 敏锐的眼光才能对语言学许多重大的理论与 实践问题作出全面的阐述与总结, 并创造性地提出系列独 到的见解。索绪尔继承了惠特尼的语言符号任意性理论, 但比惠特尼的理论体系更严密, 见解更独特, 思考得更深 远。可以这样说, 索绪尔的语言符号任意性理论是对惠特 尼理论的进一步反思与延伸, 对现代语言学理论的丰富和 发展作出了重大贡献。[3]唯有索绪尔, 才使语言研究摆脱 了长期以来服务于其它学科的附庸, 并最终确立了语言学 在人文科学中的独立地位。

\section{2. 任意性理论的现实意义}

索绪尔的任意性是其语言符号理论中的第一原则, 也 是其现代语言学理论的基石。索绪尔认为“语言最重要的 现象是思想和符号的联结”[4]（p72），这一联结是任意而 约定的; 他强调语言符号与意义之间这种任意性的普遍关 系沉积于人类大脑之中, “是人类心智和内在语言能力的 反映。种种符号之间, 种种意义之间, 都存在普遍差异。 这种普遍差异的存在具有同时性、共时性”。[5]而先前学 者是从直观的角度观察语言, 仅把语言看作命名过程中的 一种研究方法, 只有索绪尔才以一种更抽象、更系统、更 具逻辑性的思维方式来认识语言的性质, 以存在于共时态 中的有限符号蕴含无限意义的任意性理论去引导人们从
新的视角来探求语言的本质特征。因此, 正确认识索绪尔 语言符号任意性原则, 是人们了解现代语言学诞生和发展 的关键, 同时也是打开结构主义语言学大门的一把钥匙, 它开启了现代语言学研究的新天地。

\section{3. 语言符号任意性理论的局限}

\section{1. 语言符号界定存在的局限}

当然，任何理论在其发展过程中都是有其局限的，索 绪尔的理论也不例外。在《普通语言学教程》中, 索绪尔 认为语言符号所包含的两项要素都是心理的, 是由联想纽 带连接在我们的脑内, 他反对那种简单地认为名称与事物 之间存在着联系, 认为二者之间是概念和音响形象的关系。 当然, 有的学者如李葆嘉认为, 语言符号首先是事物与名 称(传统小学叫名物训诂)的关系。语言中的名物符号产生 于人能动地感知具体事物并给该事物赋予名称的活动中, 只有再把语言符号或语词加以解析, 才有音响部分与概念 部分。语言符号联系的是事物(实在的或虚幻的, 后者不 过是前者的变形)和名称, 这个事物可以化为概念移入人 脑, 但事物不等于概念, 这个名称可以变成音响形象潜入 心灵, 但名称不等于音响形象。[6]而王德春则认为任意性 与理据性的关系, 首先是语言符号与客体的联系是任意的; 其次是语言发展中语言单位之间往往具有理据性的; 再次 是理据本身也是任意的, 因而语言符号及其理据与客体都 没有必然、本质的联系。[7]我们认同李葆嘉的看法。语言 产生之前, 人们只是对事物和名称简单而任意地对应, 而 那些虚幻的事物都是从实在的事物中衍生出来的, 如“之、 所、类”等虚词都是从实词中产生的; 概念包括具体概念 和抽象概念, 而索绪尔认为语言包含的是概念和音响形象, 那就不是从语言最初的、本身的角度来分析, 这违背了他 一直坚持的原则。

索绪尔证明语言符号任意性——能指和所指的关系 是任意性的过程也是有问题的。在“语言符号任意性”中, 他提出 “姊妹” 的观念, 这在法语里用之作为能指 s-ö-r(sœur), 而其中没有任何内在关系, 它可用任何别的 音来表示。语言之间的差别或不同语言的存在就是证明: “牛”这个所指的能指在国界的一边是b- ö-f(bœuf), 另一边 却是o-k-s(Ochs)。[4]（p95-96）这毫无疑问与他一贯坚持 的原则是相矛盾的。同一概念用不同的语言去表达来证明 语言符号的任意性是毫无说服力的, 我们只需用一种语言 来说明, 挖掘语言自身所存在的根本性质即可。许国璋说: “用不同语言来说明语言的任意性, 其实是不必要的。”他 还提出：“原始时期的语言符号有任意的, 部落社会时期 是约定俗成的, 文明社会时期是立意的。”[8]许氏以语言 先为任意性, 后非任意性或者说先任意性后规约性来质疑 索绪尔的观点, 这也是难以令人信服的。索绪尔在《普通 语言学教程》中明确指出: “语言本身是任意性的, 一经 社会使用, 就具有强制性了。”[4]（p95-96）我们认为索 绪尔的这一观点是正确的。就语言符号的性质而言, 任意 性是指其单个符号最初创制时以什么样声音表达什么样 的意义, 这是任意性的; 而一旦语言符号进入交际领域, 就具有约定性了, 不能以人的意志为转移了。因此, 从社 
会来看, 不论原始社会, 还是部落社会或是文明社会时期, 所使用的语言基本上是一种经社会约定了的语言, 即使后 期社会语言有所变化, 也是原始语言的继承与发展, 语言 的内核性质不会发生根本性的改变, 不存在同一语言因社 会的发展变化而变为性质截然不同的另一种语言; 就个人 而言, 每个人使用的语言也是有强制性的, 是经过社会约 定俗成的，否则就无法交际交流思想了。

\section{2. 语言构成要素存在的局限}

\subsection{1. 语音}

语言符号连接的是概念和音响形象, 或者分别称之为 能指和所指。索绪尔认为: 能指和所指的联系是任意的, 或者, 因为我们所说的符号是指能指和所指相联结所产生 的整体，更简单地说，语言符号是任意的。[4]（p96-97） 索绪尔还指出对符号任意性这一原则可能有两种反对意 见, 一是以拟声词为依据认为能指的选择并不都是任意性 的, 列举法语的 fouet“鞭子”或glas“丧钟”等拟声词来说明 可能有一种暗示的音响刺激某些人的耳朵, 但通过追溯拉 丁语的形式来解释原来并没有这种特征, 提出当时的声音 性质只是人们赋予它们的性质, 其实这是语音演变的一种 偶然的结果, 并指出拟声词从来不是语言系统中有机组成 部分。[4]（p97-98）这种说法显然不能让人信服。例如, 汉语中牛的叫声“哞哞”, 羊的叫声“咩咩”, 水滴的声音“滴 答”，落叶的声音“沙沙”等, 自然界所发出的声音与人对 这种概念所赋予的音响形象存在惊人的相似, 如果仅仅解 释为一种惊人的巧合或一种偶然的结果, 这就明显地存在 问题了。王寅认为, 在发音方面, 发音与其所指存在着很 多自然的相似关系，如世界各种语言中的拟声词等。[9] 他以前的“从音说义”中也已提到: 全人类的语言中都有模 拟自然界物体或现象发出的声响来“取音造词”的, 它们在 音义之间自然也就有一种紧密关系, 如拟声词。[10](p28) 美国语言学家Dwight Bolinger(德怀特. 博林格)提出: 把舌 放在高和前的位置发出的元音和“小”的意义似乎存在某 种联系, 特别是wee(小的)和teeny(极小的)中的那个元音, 而那些由舌处在低的位置发出的元音总是表示“大”的意 义, 这种联系不论在哪种语言里都是存在的。[11] (p53-65) 这表明, 语音还是存有非任意性的一面。

\subsection{2. 词汇}

索绪尔为了解释派生词和复合词, 提出了绝对任意 性和相对任意性的观点。他认为, 符号任意性的基本原 则并不妨碍我们在每种语言中把根本任意的、不能论证 的同相对任意的区别开来。只有一部分符号是绝对任意 的: 别的符号中却有一种现象可以使我们看到任意性虽 不能取消, 却有程度的差别; 符号可能是相对地可以论 证的。例如, 法语的 vingt“二十”是不能论证的, 而 dix-neuf“十九”却不是在同等程度上不能论证, 因为它 会使人想起它赖以构成的要素和其他跟它有联系的要 素, 例如 dix“十”, neuf“九”, vingt-neuf"二十 九”,dix-huit“十八”, soixante-dix“七十”等等。分开来看, dix“十”和neuf“九”跟vingt“二十”一样, 但是 dix-neuf“十 九”却有相对的论证性。[4]（p177-178）对于这一点,
不少学者提出过反对意见。李葆嘉认为, 索绪尔把任意 性一分为二, 认为相对任意性即相对地可以论证, 是任 意性原则向可论证性原则复归的第一步, 把绝对任意性 解释为可论证性的转移或丧失, 是任意性原则向可论证 性原则复归的第二步, 把语言内部演化运动的标志阐述 为不断地由论证性过渡到任意性和由任意性过渡到论 证性, 则是任意性原则向可论证性原则的全面复归。[6] 我们认为李葆嘉分析得比较透彻。首先, 对复合词和派 生词的解释可以换一种方式。许多语言都有如 blackboard (黑板)、cold-blooded (冷血)、blood-pressure （血压）、football (足球)、classroom (教室)、housework (家务活) 等复合词, 还有加er或or指人的词, 如 read (阅读) 、 reader（读者）, write（写作）、writer（作 者), invent（发明）、变成inventor（发明家）, edit (编辑)、editor (编者) 等。这些复合词、派生词的 构成, 其语素都是有内在联系的, 人们大多依据经济原 则来造这些新词, 以减少记忆负担。有人还以词的曲折 变化来反对语言符号的任意性, 我们认为, 词的曲折变 化主要是语言的内部变化所致, 这与索绪尔语言符号的 任意性是无关的。其次, 从历时层次追溯语言符号的可 论性, 但其单个符号依旧是任意的, 是无法进行论证的。 例如, blackboard (黑板) 包括两个语素, 一个black（黑 色），一个board（木板）; 不管是black（黑色），还 是board (木板) , 其能指与所指都是任意性的。

\subsection{3. 句法}

句法层次方面，索绪尔仅提到非任意性，而没有论及 任意性。也就是说, 语言句法符号更多地表现出一种非任 意性。在这方面其中研究得比较深透的是沈家煊。他从认 知角度探索句法规则的约定性或语法化规律, 对句法的象 似性作了深入研究。他认为, 近年通过一批语言学家的努 力, 在语言结构特别是句法上发现了许多被人忽视的象似 性。[12]近些年对汉语句法象似性研究主要是距离象似性、 顺序象似性、数量象似性。距离象似性是指句法结构成分 的语表距离与概念之间的距离相对, 存在对应关系。如“红 糖”、“白糖”，但不会说“贵糖”，只能说“很贵的糖”, 这是 因为“红”、“白”比“贵”跟“糖”组合时距离更近。顺序象似 性指客观世界存在空间顺序、时间顺序、逻辑顺序, 汉语 的优先顺序是先参照物, 后目的物; 先发生的事情先表达, 如“他上街买菜”。数量象似性指语言形式的量和概念上的 量相对应。英语的单复数和汉语的重叠式都说明了存在数 量象似性。如汉语中的“一封信”重叠式“一封封信”、“高兴” 重叠式“高高兴兴”。不管是以韩礼德代表的系统功能语言 学还是美国功能主义语言学, 他们似乎都有很充分的证据 来证明句法的非任意性, 即句法具有相似性或理据性。目 前, 学者比较认同的——句法具有理据性。

\section{3. 语言符号与文字符号之间存在的误读}

索绪尔在《普通语言学教程》中提出: 语言和文字是 两种不同的符号系统, 后者唯一的存在理由在于表现前者。 语言学的对象不是书写的词和口说的词的结合, 而是由后 者单独构成的。但是书写的词常跟它所表现的口说的词紧 
密地混在一起, 结果篡夺了主要的作用; 人们终于把声音 符号的代表看得和这符号一样重要或比它更加重要。[4] (p85) 但索绪尔的研究只限于表音体系, 特别是以希腊 字母为原型的体系; 而汉语中的“形声字声旁却是更多地 借其自身之“形”来表义, 不是纯粹的表音符号, 更不可能 是与意义建立任意联系的记录符号”。又如, 汉字中普遍 存在的“声训”现象，也说明语音符号和意义之间的联系不 是任意性的, “凡从某声, 即有某义”意味着音义之间的关 系是固定的。实质上, “在汉语语言系统中, 声音实际与 意义相关”。[13]因此, 不少学者认为索绪尔仅研究表音体 系的文字, 而忽略了表意体系的文字; 他们还认为表意体 系的文字不具有任意性, 而具有象似性, 常以汉字来质疑 语言符号的任意性。我们认为这种观点是有问题的。首先, 文字是语言的视觉符号体系。语言产生早于文字, 语言是 通过口、耳交际的, 本质上是通过声音实现交际过程的。 但是, 由于声音一发即逝, 所以人们说话要受到时空的限 制。为了克服有声语言在时空上的限制, 人们发明了文字, 使语言除了说、听的形式以外, 又增加了写和看的形式。 文字是以书写、视觉形式对语言进行再编码的符号系统。 所以从根本上说, 语言和文字根本不是同一层面上的, 文 字也不是语言必须的组成部分, 世界上存在数千种没有文 字的语言就是一个证明。其次, 在拼音文字中, 文字是通 过字形来表达语音和语义的; 而汉字, 有人认为字形本身 就能直接表意, 不需要与语音挂钩, 可以脱离语音而存在, 这完全是错误的。尽管汉字是表意体系的文字, 易让人产 生可以“形”表“意”而不需语音参与的误解, 但汉字和其它 拼音文字一样, 是与语言音义结合的符号体系。汉字不仅 与特定的语义相关, 还同特定的语音形式紧密相联。再者, 索绪尔提出语言符号的任意性是概念和音响形象, 而文字 则是以形体表示语言符号的音义结合体。所以, 不能将语 言和文字混为一谈。

\section{4. 结论}

总而言之, 通过对语言符号任意性及语言构成要素的 分析, 我们认为索绪尔语言符号任意性观点既有它的合理 性, 也有它的局限性。诚然, 虽然索绪尔语言符号任意性 主要从其共时平面来分析论述, 受到了不少学者的质疑甚 至批评, 但是我们不能否认, “索绪尔将任意性纳入语言 学的科学体系是认识上的一场深刻变革, 虽然它在现代语 言学中还存在争论。深入讨论这样的问题, 有益于人们认 识现代语言学在理论、方法上的对立面, 有利于语言研究 的深化”。[14]从唯物辩证发展的观点看, 世界上的任何事 物都是发展变化的, 因而人们的思想认识也会随之发展变 化, 学术观点也不例外。有学者认为, 任意性从来就不是
一个有统一概念的科学术语, 语言学家使用时有着不同的 补注, 如有不可论证的、约定俗成的、非拟像的、人为的, 等等。其实, 能指和所指之间既然没有自然的联系, 但又 有许多论据支撑着能指和所指之间存在一种人为的联系, 将“人为的”和“自然的”构成一个二项对立, 倒是比较合理 的。 [8]

\section{参考文献}

[1] Bloomfield, L. 1924. Review of Saussure. Modern Language Journal8. 转引自熊兵. 美国结构主义语言学再认识 $[\mathrm{J}]$. 外 语教学与研究, 2005,(01)。

[2] 赵蓉晖. 作为社会语言学家的雅库宾斯基及其语言观[J]. 俄 语语言文学研究, 2007,(03)。

[3] 段胜峰, 李诗慧. 索绪尔语言任意性中的非任意性解读 $[J]$. 海南大学学报(人文社会科学版), 2015,(05)。

[4] 费尔迪南·德·索绪尔. 普通语言学教程[M]. 高名凯译. 北 京: 商务印书馆, 1980。

[5] 屠友祥. 语言单位: 居间介质与话语链 $[\mathrm{J}]$. 外语教学与研 究, 2016,(03)。

[6] 李葆嘉. 论索绪尔符号任意性原则的失误与复归[J]. 语言 文字应用，1994,(03)。

[7] 王德春. 论语言单位的任意性和理据性一一兼评王寅《论 语言符号象似性》 [J]. 外国语(上海外国语大学学报), 2001,(01)。

[8] 许国璋. 语言符号的任意性问题——语言哲学探索之一 [J]. 外语教学与研究, 1988,(03)。

[9] 王寅. 论语言符号象似性 [J]. 外语与外语教学, 1999,(05)。

[10] 王寅、李弘. 英语词汇速记教程[M]. 北京: 科学普及出版 社, 1996。

[11] Dwight Bolinger. Aspects of Language[M], 方立等译. 北京: 外语教学与研究出版社, 1999。

[12] 沈家煊. 句法的象似性问题 [J]. 外语教学与研究, 1993,(01)。

[13] 李川. 二分与三合: 从言一文角度看中西思维方式的分野 $[\mathrm{J}]$. 郑州大学学报(哲社科版), 2016,(02)。

[14] 王红生. 语言符号的任意性及其理论效能 $[\mathrm{J}]$. 西安交通大 学学报(社科版), 2017,(03)。 